\title{
On class invariants for non-holomorphic modular functions and a question of Bruinier and Ono
}

Michael H Mertens ${ }^{*}$ and Larry Rolen ${ }^{\dagger}$

\section{${ }^{*}$ Correspondence:}

mmertens@math.uni-koeln.de

${ }^{\dagger}$ Equal contributor

Mathematisches Institut der

Universität zu Köln, Weyertal 86-90,

D-50931 Köln, Germany

\begin{abstract}
Recently, Bruinier and Ono found an algebraic formula for the partition function in terms of traces of singular moduli of a certain non-holomorphic modular function. In this paper we prove that the rational polynomial having these singular moduli as zeros is (essentially) irreducible, settling a question of Bruinier and Ono. The proof uses careful analytic estimates together with some related work of Dewar and Murty, as well as extensive numerical calculations of Sutherland.
\end{abstract}

\section{Background}

A partition of a natural number $n$ is a non-increasing sequence of natural numbers which sum up to $n$. Let $p(n)$ denote the number of partitions of $n$. Despite its elementary definition, it is computationally infeasible to compute this number directly for large $n$. A much more efficient method for computing the partition number was offered by Euler's recursive formula for $p(n)$, obtained as a consequence of his pentagonal number theorem [6]. Much later, Hardy and Ramanujan [11] found the asymptotic formula

$$
p(n) \sim \frac{1}{4 n \sqrt{3}} e^{\pi \sqrt{\frac{2 n}{3}}} \text { as } n \rightarrow \infty,
$$

developing and employing a device which has become essential in analytic number theory, the so-called Circle Method. By refining their method, Rademacher [20] found his famous exact formula for $p(n)$,

$$
p(n)=\frac{2 \pi}{(24 n-1)^{\frac{3}{4}}} \sum_{k=1}^{\infty} \frac{A_{k}(n)}{k} I_{\frac{3}{2}}\left(\frac{\pi \sqrt{24 n-1}}{6 k}\right),
$$

where $I_{\frac{3}{2}}$ is the modified Bessel function of the first kind and $A_{k}(n)$ is a certain Kloosterman sum.

More recently, Bruinier and Ono [3] proved that $p(n)$ can also be computed as a finite sum of distinguished algebraic numbers. More precisely, they showed that

$$
p(n)=\frac{1}{24 n-1} \sum_{Q \in \mathcal{Q}_{\delta}} P\left(\tau_{Q}\right),
$$

(C) 2015 Mertens and Rolen; licensee Springer. This is an Open Access article distributed under the terms of the Creative Commons Attribution License (http://creativecommons.org/licenses/by/4.0), which permits unrestricted use, distribution, and reproduction in any medium, provided the original work is properly credited. 
where $\mathcal{Q}_{\delta}$ is a set of representatives of positive definite binary integral quadratic forms $Q(x, y)=a x^{2}+b x y+c y^{2}$ of discriminant $\delta:=-24 n+1$ satisfying $6 \mid a$, modulo the action of the group $\Gamma_{0}(6)$, where we choose a set of representatives such that $b \equiv 1(\bmod 12)$. Moreover, $\tau_{Q}$ is the unique point in the upper half-plane $\mathbb{H}$ satisfying $Q\left(\tau_{Q}, 1\right)=0$, and $P$ is a certain Maass form of weight 0 defined in (2.2).

In particular, Bruinier and Ono proved that $P\left(\tau_{Q}\right)$ is an algebraic number, and in fact it is known that $(24 n-1) P\left(\tau_{Q}\right)$ is an algebraic integer [16]. For related work studying and applying formulas for the partition function in terms of traces of singular moduli, the interested reader is also referred to $[2,8,15]$. In this context, it is natural to define the polynomial

$$
H_{\delta}(x):=\prod_{Q \in \mathcal{Q}_{\delta}}\left(x-P\left(\tau_{Q}\right)\right) \in \mathbb{Q}[x] .
$$

By an elementary calculation (see Lemma 3.7 of [4]), this factors over $\mathbb{Q}$ as

$$
H_{\delta}(x)=\prod_{\substack{f>0 \\ f^{2} \mid \delta}} \varepsilon(f)^{h\left(\frac{\delta}{f^{2}}\right)} \widehat{H}_{\frac{\delta}{f^{2}}}(\varepsilon(f) x),
$$

where $h(d)$ denotes the class number of discriminant $d, \varepsilon(f)=1$ if $f \equiv \pm 1(\bmod 12)$ and $\varepsilon(f)=-1$ otherwise. We also set

$$
\widehat{H}_{\delta}(x):=\prod_{Q \in \mathcal{P}_{\delta}}\left(x-P\left(\tau_{Q}\right)\right)
$$

where $\mathcal{P}_{\delta}$ is the set of primitive forms in $\mathcal{Q}_{\delta}$, i.e., those forms for which $\operatorname{gcd}(a, b, c)=1$.

Bruinier and Ono [3] asked whether $\widehat{H}_{\delta}(x)$ is irreducible and in [4] they produced, together with Sutherland, very strong numerical evidence for the affirmative answer. In this paper, we settle their question.

Theorem 1.1. The polynomial $\widehat{H}_{\delta}(x)$ is irreducible over $\mathbb{Q}$. Moreover we have

$$
\Omega_{t} \cong K[x] /\left(\widehat{H}_{\delta}(x)\right)
$$

where $\delta=-24 n+1=t^{2} d$ with $d$ a fundamental discriminant and $\Omega_{t}$ the ring class field of the order of conductor $t$ in $K:=\mathbb{Q}(\sqrt{d})$.

Remark. It should be possible to give a general version of Theorem 1.1 for almost holomorphic modular functions with rational expansions at the cusps, however, the authors have chosen to highlight this special case for the sake of explicitness, and in particular in order to establish the irreducibility for all polynomials, as opposed to all but finitely many in the general case.

The paper is organized as follows. In Section 2 we recall some tools required for the proof of Theorem 1.1 such as Masser's formula and a convenient form of Shimura reciprocity due to Schertz. The proof itself is the subject of Section 3. 


\section{Preliminaries}

\subsection{Masser's formula}

Throughout, $\tau=u+i v \in \mathbb{H}$ (the complex upper half-plane) with $u, v \in \mathbb{R}$, and let $q:=e^{2 \pi i \tau}$. For a function $f: \mathbb{H} \rightarrow \mathbb{C}$, an integer $k$, and a matrix $\gamma=\left(\begin{array}{ll}a & b \\ c & d\end{array}\right) \in \mathrm{GL}_{2}(\mathbb{R})$ with $\operatorname{det} \gamma>0$ we define the weight $k$ slash operator by

$$
\left.f\right|_{k} \gamma(\tau):=\operatorname{det}(\gamma)^{\frac{k}{2}}(c \tau+d)^{-k} f\left(\frac{a \tau+b}{c \tau+d}\right) .
$$

Denote by $E_{k}$ the normalized weight $k$ Eisenstein series for $\mathrm{SL}_{2}(\mathbb{Z})$ with leading coefficient 1 , and let

$$
j(\tau):=1728 \frac{E_{4}^{3}(\tau)}{E_{4}^{3}(\tau)-E_{6}^{2}(\tau)}
$$

be the classical $j$-invariant. Furthermore,

$$
\eta(\tau):=q^{\frac{1}{24}} \prod_{\ell=1}^{\infty}\left(1-q^{\ell}\right)
$$

is the Dedekind eta function. We require the function

$$
F(\tau):=\frac{E_{2}(\tau)-2 E_{2}(2 \tau)-3 E_{2}(3 \tau)+6 E_{2}(6 \tau)}{2 \eta(\tau)^{2} \eta(2 \tau)^{2} \eta(3 \tau)^{2} \eta(6 \tau)^{2}}=q^{-1}-10-29 q-104 q^{2}-\ldots,
$$

which is a weakly holomorphic modular form of weight -2 for $\Gamma_{0}(6)$. The Maass raising operator

$$
R_{k}:=\frac{1}{2 \pi i} \frac{\partial}{\partial \tau}-\frac{k}{4 \pi v}
$$

maps this weakly holomorphic modular form $F$ to a non-holomorphic modular function

$$
P(\tau):=-R_{-2}(F)(\tau)
$$

which is an eigenfunction of the hyperbolic Laplacian

$$
\Delta:=-v^{2}\left(\frac{\partial^{2}}{\partial u^{2}}+\frac{\partial^{2}}{\partial v^{2}}\right)
$$

with eigenvalue -2 .

As in [16], we decompose $P$ as

$$
P=A+B \cdot C
$$

where $A$ and $B$ are modular functions for $\Gamma_{0}(6)$ given by

$$
\begin{aligned}
A(\tau) & :=-\frac{1}{2 \pi i} \cdot \frac{\partial}{\partial \tau} F(\tau)-\frac{1}{6} E_{2}(\tau) F(\tau)+\frac{F(\tau) E_{6}(\tau)(7 j(\tau)-6912)}{6 E_{4}(\tau)(j(\tau)-1728)}, \\
B(\tau) & :=\frac{F(\tau) E_{6}(\tau) j(\tau)}{E_{4}(\tau)},
\end{aligned}
$$

and $C$ is a non-holomorphic modular function for $\mathrm{SL}_{2}(\mathbb{Z})$, given by

$$
C(\tau):=\frac{E_{4}(\tau)}{6 E_{6}(\tau) j(\tau)}\left(E_{2}(\tau)-\frac{3}{\pi v}\right)-\frac{7 j(\tau)-6912}{6 j(\tau)(j(\tau)-1728)} .
$$


This decomposition is especially useful as Masser gives a very important formula for the singular moduli of $C$ in Appendix I in [18]. To state this result, we recall the modular polynomial $\Phi_{-D}(x, y)$ which is defined for a discriminant $D<0$ by the relation

$$
\Phi_{-D}(j(\tau), y):=\prod_{M \in \mathcal{V}}(y-j(M \tau)) .
$$

Here, $\mathcal{V}$ is a system of representatives of $\mathrm{SL}_{2}(\mathbb{Z}) \backslash \Gamma_{-D}$, where $\Gamma_{-D}$ denotes the set of all primitive integral $2 \times 2$-matrices of determinant $-D$ (see e.g. [13], Section IV.1.6). It is a well-known fact (cf. [14], Chapter 5, Section 2) that $\Phi_{-D}(x, y) \in \mathbb{Z}[x, y]$. Now let $Q$ be a quadratic form ${ }^{\mathrm{a}}$ of discriminant $D$ and $\tau_{Q}$ the corresponding $C M$-point. Then we define numbers $\beta_{\mu, v}\left(\tau_{Q}\right)$ via the expansion

$$
\Phi_{-D}(x, y)=: \sum_{\mu, v} \beta_{\mu, v}\left(\tau_{Q}\right)\left(x-j\left(\tau_{Q}\right)\right)^{\mu}\left(y-j\left(\tau_{Q}\right)\right)^{\nu} .
$$

It is easy to see that all these numbers lie in the field $\mathbb{Q}\left(j\left(\tau_{Q}\right)\right)$ and that $\beta_{\mu, \nu}\left(\tau_{Q}\right)=$ $\beta_{v, \mu}\left(\tau_{Q}\right)$. Masser's formula then states that in the case that the discriminant $D$ is not special, i.e., not of the form $D=-3 d^{2}$, one has

$$
C\left(\tau_{Q}\right)=\frac{2 \beta_{0,2}\left(\tau_{Q}\right)-\beta_{1,1}\left(\tau_{Q}\right)}{\beta_{1,0}\left(\tau_{Q}\right)} .
$$

Note in particular that for any $n, \delta=-24 n+1$ is not special. Using the formula of Masser, we are able to reduce our problem of studying singular moduli for nonholomorphic modular functions to the study of holomorphic modular functions associated to each discriminant, as in the following result.

Lemma 2.1. For every non-special discriminant $D<0$, there exists a (meromorphic) modular function $M_{D}$ for $\Gamma_{0}(6)$ such that

$$
P\left(\tau_{Q}\right)=M_{D}\left(\tau_{Q}\right)
$$

for all quadratic forms $Q$ of discriminant $D$.

Proof. Using the definition of the modular polynomial one finds explicitly that (see also (2.9) in [4])

$$
\begin{aligned}
& \beta_{0,1}\left(\tau_{Q}\right)=[y] \Phi_{-D}\left(j\left(\tau_{Q}\right), y+j\left(\tau_{Q}\right)\right), \\
& \beta_{1,1}\left(\tau_{Q}\right)=[y] \Phi_{-D}^{\prime}\left(j\left(\tau_{Q}\right), y+j\left(\tau_{Q}\right)\right), \\
& \beta_{0,2}\left(\tau_{Q}\right)=\left[y^{2}\right] \Phi_{-D}\left(j\left(\tau_{Q}\right), y+j\left(\tau_{Q}\right)\right),
\end{aligned}
$$

where $\Phi_{-D}^{\prime}:=\frac{\partial}{\partial x} \Phi_{-D}$ and $\left[y^{k}\right] \Phi_{-D}(x, y)$ denotes the coefficient of $y^{k}$ in $\Phi_{-D}$. The righthand sides of (2.7)-(2.9) clearly also make sense if we replace the algebraic number $j\left(\tau_{Q}\right)$ by the modular function $j(\tau)$, yielding modular functions $\beta_{\mu, v}(\tau)$ for $\mathrm{SL}_{2}(\mathbb{Z})$. Thus the function

$$
M_{D}(\tau):=A(\tau)+B(\tau) \frac{2 \beta_{0,2}(\tau)-\beta_{1,1}(\tau)}{\beta_{1,0}(\tau)}
$$

has the desired properties. 


\section{$2.2 N$-systems}

In this subsection, we cite some results of Schertz [22]. We begin by recalling a convenient set of representatives for primitive quadratic forms introduced in [22] for the study of class invariants known as Weber's class invariants.

Definition 2.2. Let $N \in \mathbb{N}$ and $D=t^{2} d<0$ be a discriminant, with $t \in \mathbb{N}$ and $d a$ fundamental discriminant. Moreover, let $\left\{Q_{1}, \ldots, Q_{r}\right\}\left(Q_{j}(x, y)=a_{j} x^{2}+b_{j} x y+c_{j} y^{2}\right)$ be a system of representatives of primitive quadratic forms modulo $\mathrm{SL}_{2}(\mathbb{Z})$. We call the set $\left\{Q_{1}, \ldots, Q_{r}\right\}$ an $N$-system mod $t$ if the conditions

$$
\operatorname{gcd}\left(c_{j}, N\right)=1 \quad \text { and } \quad b_{j} \equiv b_{\ell}(\bmod N), 1 \leq j, \ell \leq r
$$

are satisfied.

Remark. Schertz gave this definition (see [22], p. 329) in terms of ideal classes of the ring class field $\Omega_{t}$ and with switched roles of a and $c$. He also proved constructively that an $N$-system mod t always exists (see [22], Proposition 3).

The following theorem (see [22], Theorem 4) is a key in the proof of Theorem 1.1.

Theorem 2.3 (Schertz). Let $g$ be a modular function for $\Gamma_{0}(N)$ for some $N \in \mathbb{N}$ whose Fourier coefficients at all cusps lie in the Nth cyclotomic field. Suppose furthermore that $g(\tau)$ and $g\left(-\frac{1}{\tau}\right)$ have rational Fourier coefficients, and let $Q(x, y)=a x^{2}+b x y+c y^{2}$ be a quadratic form of discriminant $D=t^{2} d, d$ a fundamental discriminant, with $\operatorname{gcd}(c, N)=$ 1 and $N \mid a$. Then we have that $g\left(\tau_{Q}\right) \in \Omega_{t}$ unless $g$ has a pole at $\tau_{Q}$.

Moreover, if $\left\{Q=Q_{1}, \ldots, Q_{r}\right\}$ is an $N$-system $\bmod t$, then

$$
\left\{g\left(\tau_{Q_{1}}\right), \ldots, g\left(\tau_{Q_{r}}\right)\right\}=\left\{\sigma\left(g\left(\tau_{Q_{1}}\right)\right): \sigma \in \mathrm{Gal}_{D}\right\}
$$

where Gal $_{D}$ denotes the Galois group of $\Omega_{t} / \mathbb{Q}(\sqrt{d})$.

Remark. Schertz stated the above theorem for modular functions for the group

$$
\Gamma^{0}(N):=\left\{\left(\begin{array}{ll}
a & b \\
c & d
\end{array}\right) \in \mathrm{SL}_{2}(\mathbb{Z}): b \equiv 0(\bmod N)\right\}
$$

which is clearly isomorphic to $\Gamma_{0}(N)$ via conjugation with $S:=\left(\begin{array}{rr}0 & -1 \\ 1 & 0\end{array}\right)$. This conjugation must be carried over to the quadratic forms as well which explains the change of roles of the coefficients a and c compared to [22].

\subsection{Poincaré series}

In this subsection we briefly recall some important facts about Maass-Poincaré series. For more details, we refer to the survey in Section 8.3 of [19] and the earlier works $[7,12]$.

Let $M_{\nu, \mu}$ denote the usual $M$-Whittaker function (see e.g. [10], p. 1014) and define for $v>0, k \in \mathbb{Z}$, and $s \in \mathbb{C}$ the function

$$
\mathcal{M}_{s, k}(v):=v^{-\frac{k}{2}} M_{-\frac{k}{2}, s-\frac{1}{2}}(v)
$$


Using this, we construct the following Poincaré series for $\Gamma_{0}(N)$,

$$
P_{m, s, k, N}(\tau):=\left.\frac{1}{2 \Gamma(2 s)} \sum_{\gamma \in \Gamma_{\infty} \backslash \Gamma_{0}(N)}\left[\mathcal{M}_{s, k}(4 \pi m v) e^{-2 \pi i m u}\right]\right|_{k} \gamma
$$

where $m \in \mathbb{N}, \tau=u+i v \in \mathbb{H}, \operatorname{Re}(s)>1, k \in-\mathbb{N}$, and $\Gamma_{\infty}:=\left\{ \pm\left(\begin{array}{ll}1 & n \\ 0 & 1\end{array}\right): n \in \mathbb{Z}\right\}$. It is not hard to check (see e.g. Proposition 2.2 in [3]) that under the Maass raising operator, the Poincaré series $P_{m, s, k, N}$ is again mapped to a Poincaré series

$$
R_{k}\left(P_{m, s, k, N}\right)=m\left(s+\frac{k}{2}\right) P_{m, s, k+2, N} .
$$

In the special case when $k<0$ and $s=1-\frac{k}{2}$, the series $P_{m, k, N}:=P_{m, 1-\frac{k}{2}, k, N}$ defines a harmonic Maass form of weight $k$ for $\Gamma_{0}(N)$ whose principal part at the cusp $\infty$ is given by $q^{-m}$, and at all the other cusps the principal part is 0 . In this situation, we have the following explicit Fourier expansion at $\infty$ (see e.g. [19], Theorem 8.4).

Proposition 2.4. For $m, N \in \mathbb{N}, k \in-\mathbb{N}$, and $\tau \in \mathbb{H}$ we have

$$
(1-k) ! P_{m, k, N}(\tau)=(k-1)(\Gamma(1-k, 4 \pi m v)-\Gamma(1-k)) q^{-m}+\sum_{\ell \in \mathbb{Z}} b_{m, k, N}(\ell, v) q^{\ell},
$$

where the coefficients $b_{m, k, N}(\ell, v)$ are defined as follows.

1) If $\ell<0$, then

$$
\begin{aligned}
b_{m, k, N}(\ell, v)= & 2 \pi i^{2-k}(k-1) \Gamma(1-k, 4 \pi|\ell| v)\left|\frac{\ell}{m}\right|^{\frac{k-1}{2}} \\
& \times \sum_{\substack{c>0 \\
c \equiv 0(\bmod N)}} \frac{K(-m, \ell, c)}{c} \cdot J_{1-k}\left(\frac{4 \pi \sqrt{|m \ell|}}{c}\right),
\end{aligned}
$$

where

$$
\Gamma(\alpha ; x):=\int_{x}^{\infty} e^{-t} t^{\alpha-1} d t .
$$

2) If $\ell>0$, then

$$
b_{m, k, N}(\ell, v)=-2 \pi i^{2-k}(1-k) ! \ell^{\frac{k-1}{2}} m^{\frac{1-k}{2}} \sum_{\substack{c>0 \\ c \equiv 0(\bmod N)}} \frac{K(-m, \ell, c)}{c} \cdot I_{1-k}\left(\frac{4 \pi \sqrt{|m \ell|}}{c}\right) .
$$

3) If $\ell=0$, then

$$
b_{m, k, N}(0, v)=-(2 \pi i)^{2-k} m^{1-k} \sum_{\substack{c>0 \\ c \equiv 0(\bmod N)}} \frac{K(-m, 0, c)}{c^{2-k}} .
$$

Here, $I_{s}$ and $J_{s}$ denote the usual $I$ - and J-Bessel functions and $K(m, \ell, c)$ is the usual Kloosterman sum,

$$
K(m, \ell, c):=\sum_{d(\bmod c)^{*}} \exp \left(2 \pi i\left(\frac{m \bar{d}+\ell d}{c}\right)\right),
$$

where $d$ runs through the residue classes $(\bmod c)$ which are coprime to $c$ and $\bar{d}$ denotes the multiplicative inverse of $d(\bmod c)$. 


\section{Irreducibility of $\widehat{H}_{\delta}(x)$}

Now that we have recalled the relevant known facts, we proceed towards proving our main result, Theorem 1.1. We require some information about the Fourier expansions of $A, B$, and $M_{D}$, from Equations (2.3), (2.4), and (2.10) respectively, at all cusps, which we provide in the following lemma.

Lemma 3.1. 1. The modular functions $A$ and $B$ have Fourier expansions with rational Fourier coefficients at all cusps of $\Gamma_{0}(6)$.

2. If $D<0$ is a non-special discriminant, then the Fourier coefficients of $M_{D}$ at all cusps lie in the field $\mathbb{Q}\left(\zeta_{6}\right)$, where $\zeta_{6}:=e^{\frac{2 \pi i}{6}}$, and the Fourier coefficients of $M_{D}(\tau)$ and $M_{D}\left(-\frac{1}{\tau}\right)$ are rational.

Proof. (1) The denominator of the function $F$ lies in the one-dimensional space $S_{4}\left(\Gamma_{0}(6)\right)$, and hence is an eigenfunction of all Atkin-Lehner operators, the eigenvalue being always 1 . The numerator, which we denote by $F_{1}$, is a weight two modular form for $\Gamma_{0}(6)$ and is an eigenfunction of all Atkin-Lehner involutions as well. This can be seen by a short and direct calculation:

For the convenience of the reader, we give some details. Namely, note that the AtkinLehner operators obtained by slashing with one of the following matrices

$$
W_{6}:=\left(\begin{array}{rr}
0 & -1 \\
6 & 0
\end{array}\right), W_{3}:=\left(\begin{array}{ll}
3 & 1 \\
6 & 3
\end{array}\right), W_{2}:=\left(\begin{array}{ll}
2 & -1 \\
6 & -2
\end{array}\right),
$$

map the cusp $\infty$ to $0, \frac{1}{2}, \frac{1}{3}$, (respectively).

One finds by a direct calculation of the Smith normal form of the matrices $W_{d}, d=$ $2,3,6$, that

$$
\left.F_{1}\right|_{2} W_{6}=F_{1},\left.\quad F_{1}\right|_{2} W_{3}=-F_{1}, \quad \text { and }\left.F_{1}\right|_{2} W_{2}=-F_{1} .
$$

Therefore, $F$ is an eigenfunction of all Atkin-Lehner involutions of level 6.

Using this,we can directly calculate the Fourier expansion of $F$ at all cusps (correcting a typo in (3.2) of [5]). To this end we choose the following 12 right coset representatives of $\mathrm{SL}_{2}(\mathbb{Z}) / \Gamma_{0}(6)$ (where $\left.T:=\left(\begin{array}{ll}1 & 1 \\ 0 & 1\end{array}\right)\right)$,

$$
\begin{array}{ll}
\gamma_{\infty}:=\left(\begin{array}{ll}
1 & 0 \\
0 & 1
\end{array}\right), & \\
\gamma_{\frac{1}{3}, r}:=\left(\begin{array}{ll}
1 & 0 \\
3 & 1
\end{array}\right) T^{r} \quad \text { for } r=0,1, \\
\gamma_{\frac{1}{2}, s}:=\left(\begin{array}{ll}
1 & 1 \\
2 & 3
\end{array}\right) T^{s} \quad \text { for } s=0,1,2, \\
\gamma_{0, t}:=\left(\begin{array}{rr}
0 & -1 \\
1 & 0
\end{array}\right) T^{t} \quad \text { for } t=0,1,2,3,4,5 .
\end{array}
$$

Using the relation $W_{6}=\gamma_{0,0} V_{6}$, where $V_{d}:=\left(\begin{array}{ll}d & 0 \\ 0 & 1\end{array}\right)$, we find that

$$
F(\tau)=F\left|2 W_{6}(\tau)=\frac{1}{6} F\right|_{2} \gamma_{0,0}(6 \tau)
$$


Thus we find the following Fourier expansions of $F$ at the cusp 0 ,

$$
\left.F\right|_{2} \gamma_{0, t}(\tau)=6 \sum_{m=-1}^{\infty} a_{m} \zeta_{6}^{t m} q^{\frac{m}{6}}
$$

where $F(\tau)=: \sum_{m=-1}^{\infty} a_{m} q^{m}$. Similarly, we have

$$
W_{2}=\gamma_{0,0} V_{2} \gamma_{\frac{1}{2}, 0}^{-1}, \quad W_{3}=\gamma_{0,0}^{-1} V_{3} T^{-1} \gamma_{\frac{1}{3}, 0}^{-1}\left(\begin{array}{ll}
1 & 0 \\
6 & 1
\end{array}\right),
$$

which yields

$$
F(\tau)=-\left.F\right|_{2} W_{2}(\tau)=-\left.6 F\right|_{2} V_{2} \gamma_{\frac{1}{2}, 0}^{-1}\left(\frac{\tau}{6}\right)=-\left.3 F\right|_{2} \gamma_{\frac{1}{2}, 0}\left(\frac{\tau}{3}\right) .
$$

Hence, at $\frac{1}{2}$, we have the Fourier expansions

$$
\left.F\right|_{2 \gamma_{\frac{1}{2}, s}}(\tau)=3 \sum_{m=-1}^{\infty} a_{m} \zeta_{6}^{3+2 m s} q^{\frac{m}{3}} .
$$

A similar calculation yields the Fourier expansions at $\frac{1}{3}$ :

$$
\left.F\right|_{2} \gamma_{\frac{1}{3}, r}(\tau)=2 \sum_{m=-1}^{\infty} a_{m}(-1)^{m r} q^{\frac{m}{2}} .
$$

Thus, it is clear that $B$ has a Fourier expansion with rational coefficients at all cusps, since $\frac{B}{F}$ is a weight 2 meromorphic modular form for $\mathrm{SL}_{2}(\mathbb{Z})$ which has rational (in fact integral) Fourier coefficients at $\infty$.

The Serre derivative of $F$, which is given by $\frac{1}{2 \pi i} \frac{\partial}{\partial \tau} F(\tau)+\frac{1}{6} E_{2}(\tau) F(\tau)=: A_{1}(\tau)$ has a rational leading term at $\infty$, namely $\frac{7}{6}$, and integer coefficients otherwise (note that for $n>0$ the $n$-th coefficient of $E_{2}$ is divisible by 24). The same is true for the function

$$
A_{2}(\tau):=\frac{F(\tau) E_{6}(\tau)(7 j(\tau)-6912)}{6 E_{4}(\tau)(j(\tau)-1728)}
$$

(again, note that all but the zeroth Fourier coefficient of $E_{6}$ are divisible by $504=6$. 84). Therefore, $A=-A_{1}+A_{2}$, has integer coefficients at $\infty$. Since the Serre derivative commutes with the action of $\mathrm{SL}_{2}(\mathbb{Z})$ and $A_{2}$ is the product of $F$ and a level 1 form, the same argument as for $B$ yields the claim.

(2) It remains to show that the level 1 modular function

$$
\frac{2 \beta_{0,2}(\tau)-\beta_{1,1}(\tau)}{\beta_{1,0}(\tau)}
$$

from (2.10) has rational Fourier coefficients at $\infty$. But since by definition the functions $\beta_{\mu, v}(\tau)$ are polynomials in $j(\tau)$ with rational coefficients, this is clear.

With this, we can immediately show the following.

Proposition 3.2. Let $P$ be as in as in the introduction, $\delta=-24 n+1=t^{2} d$ with $d a$ fundamental discriminant. Then $P\left(\tau_{Q_{0}}\right) \in \Omega_{t}$ and

$$
\left\{P\left(\tau_{Q}\right): Q \in \mathcal{P}_{\delta}\right\}=\left\{\sigma\left(P\left(\tau_{Q_{0}}\right)\right): \sigma \in \mathrm{Gal}_{\delta}\right\}
$$


for all $Q_{0} \in \mathcal{P}_{\delta}$. In particular, the values $P\left(\tau_{Q}\right)$ for $Q \in \mathcal{P}_{\delta}$ generate the ring class field $\Omega_{t}$ over $\mathbb{Q}(\sqrt{d})$ and $\widehat{H}_{\delta}(x)$ is a perfect power of an irreducible polynomial.

Proof. By the proposition on p. 505 of [9], we know that the set $\mathcal{P}_{\delta}$ also represents the $\mathrm{SL}_{2}(\mathbb{Z})$-equivalence classes of primitive quadratic forms of discriminant $\delta$. Hence $\mathcal{P}_{\delta}$ is a 6-system $\bmod t$, and by Lemma 2.1 we know that for all $Q \in \mathcal{P}_{\delta}$ we have $P\left(\tau_{Q}\right)=$ $M_{\delta}\left(\tau_{Q}\right)$ for some modular function $M_{\delta}$ for $\Gamma_{0}(6)$. By Lemma 3.1, this function satisfies the conditions in Theorem 2.3, which immediately gives the first part of the proposition. The second part follows from elementary Galois theory and the fact that any finite extension of $\mathbb{Q}(\sqrt{d})$ is separable.

The remainder of this section is devoted to the proof that $\widehat{H}_{\delta}$ is itself an irreducible polynomial. By Proposition 3.2, this is equivalent to showing that there is at least one singular modulus $P\left(\tau_{Q}\right)$ which is assumed only once as $Q$ ranges over $\mathcal{P}_{\delta}$. To see this, we use an effective version of Lemma 5 in [5]. For this, let

$$
\begin{aligned}
& \mathcal{F}:=\left\{\tau \in \mathbb{H}:-\frac{1}{2}<\operatorname{Re}(\tau)\right.\left.\leq \frac{1}{2} \text { and }|\tau|>1\right\} \\
& \cup\left\{\tau \in \mathbb{H}:|\tau|=1 \text { and } 0 \leq \operatorname{Re}(\tau) \leq \frac{1}{2}\right\}
\end{aligned}
$$

denote the usual fundamental domain for the action of $\mathrm{SL}_{2}(\mathbb{Z})$ on $\mathbb{H}$. Our main analytic result is then the following, which is an effective form of a result of Dewar and Murty (see Lemma 5 in [5]).

Lemma 3.3. Let $\gamma$ be one of the 12 matrices in (3.1) and assume that

$$
\left.F\right|_{-2} \gamma(\tau)=h \zeta q^{-\frac{1}{h}}+a_{0}+a_{1} q^{\frac{1}{h}}+\ldots,
$$

where $h$ is the width of the cusp $\gamma \infty$ and $\zeta$ is a certain 6 th root of unity. Then, for every $\gamma$ and $\tau \in \mathcal{F}$, we have

$$
\left.P\right|_{0} \gamma(\tau)=\zeta\left(1-\frac{h}{2 \pi \nu}\right) e^{-\frac{2 \pi i \tau}{h}}+E_{\gamma}(\tau),
$$

where the error $E_{\gamma}$ satisfies the uniform bound $\left|E_{\gamma}(\tau)\right| \leq \kappa:=1334.42$.

Proof. We can express the function $F$ in terms of Poincaré series via

$$
F=P_{1,-2,6}-\left.P_{1,-2,6}\right|_{-2} W_{2}-\left.P_{1,-2,6}\right|_{-2} W_{3}+\left.P_{1,-2,6}\right|_{-2} W_{6},
$$

(see [3], p. 213). Then note that $\left|P_{1,-2,6}\right|_{-2} W_{d} \mid, d=1,2,3,6$ is majorized by

$$
\left|q^{-1}\right|+\sum_{\ell \in \mathbb{Z}}\left|b_{1,-2,6}(\ell, v)\right| \cdot|q|^{\ell}
$$

with $b_{1,-2,6}(\ell, v)$ as given in Proposition 2.4. Indeed, the Maass-Poincaré series, defined in Section 2.3, grow exponentially approaching the cusp $\infty$ and have moderate growth at the other cusps. Following [21] (see in particular Chapter 5 there), the Bringmann and Ono [1] introduced Maass-Poincaré series which grow exponentially at any given cusp and grow moderately at all the others. In [21], Theorem 5.1.2., the function $\left.\widetilde{P}\right|_{0} \sigma$ with $\widetilde{P}$ a Poincaré series for a group $\Gamma$ and $\sigma \in \mathrm{GL}_{2}(\mathbb{Q})$ with $\operatorname{det}(\sigma)>0$ is expressed in terms of a Poincaré series for the group $\sigma^{-1} \Gamma \sigma$, possibly growing at a different cusp, see also Remark 3.4 below. The explicit Fourier expansions of these series, given in Theorem 3.2 of [1], easily 
implies our claim because the Atkin-Lehner operators $W_{d}$ normalize $\Gamma_{0}(6)$. Futhermore, we can ignore the non-holomorphic parts of the Maass-Poincaré series because $F$ is holomorphic on $\mathbb{H}$, and thus they must cancel.

Thus, we are left to bound the Fourier coefficients of the Poincare series $P_{1,-2,6}(\tau)=$ $q^{-1}+\frac{1}{6} \sum_{\ell \in \mathbb{Z}} b_{1,-2,6}(\ell, v) q^{\ell}$ (for $\ell \geq 0$ ) from above to obtain an upper bound for our error function $E_{\gamma}$. Note that our estimates could be improved, but for the purpose of this paper, we do not require this. Furthermore, we make all constants explicit, which is crucial for our purposes in order to obtain the absolute bound $\kappa$. Clearly, we may estimate

$$
|K(m, \ell, c)| \leq c
$$

Furthermore, for $x>0$ and $v>-\frac{1}{2}$ we have the bound (eq. (6.25) in [17])

$$
I_{v}(x)<\frac{1}{\Gamma(v+1)}\left(\frac{x}{2}\right)^{v} \cosh (x)
$$

which, for $0<x \leq 1$, implies

$$
I_{v}(x) \leq \frac{2}{\Gamma(v+1)}\left(\frac{x}{2}\right)^{v}
$$

For $x \geq 1$, we can bound

$$
I_{v}(x) \leq \frac{e^{x}}{\sqrt{2 \pi x}}
$$

which follows from the well-known asymptotic formula for $I_{v}$ (see e.g. [10], eq. 8.451.5). We now split the expression for $b_{1,-2,6}(\ell, v)$ in Proposition 2.4 and use the above bounds (3.5), (3.7), (3.8) to obtain for $\ell \in \mathbb{N}$ that

$$
\begin{aligned}
\left|b_{1,-2,6}(\ell, v)\right| & \leq 12 \pi \ell^{-\frac{3}{2}}\left[\frac{2 \pi \sqrt{\ell}}{3} \frac{\exp \left(\frac{2 \pi \sqrt{\ell}}{3}\right)}{2 \pi \sqrt{\frac{\sqrt{\ell}}{3}}}+\sum_{c>\frac{2 \pi \sqrt{\ell}}{3}} \frac{1}{3}\left(\frac{\pi \sqrt{\ell}}{3 c}\right)^{3}\right] \\
& \leq 12 \pi \ell^{-\frac{3}{2}}\left[\frac{\ell^{\frac{1}{4}}}{\sqrt{3}} \exp \left(\frac{2 \pi \sqrt{\ell}}{3}\right)+\frac{\pi^{3}}{81} \ell^{\frac{3}{2}} \zeta(3)\right] .
\end{aligned}
$$

Furthermore, it follows directly that

$$
\left|b_{1,-2,6}(0, v)\right| \leq \frac{2}{27} \pi^{4} \zeta(3)
$$

Now let $\tau \in \mathcal{F}$, which, in particular, implies that $v \geq \frac{\sqrt{3}}{2}$, and $\gamma$ one of the matrices in (3.1). Then, as applying the matrix $\gamma$ to $F$ has the effect of multiplying its absolute value by the width $h$ of the cusp $\gamma \infty$, we find that 


$$
\begin{aligned}
& \left|E_{\gamma}(\tau)\right|=|P|_{0} \gamma(\tau)-\zeta\left(1-\frac{h}{2 \pi v}\right) q^{-\frac{1}{h}}|=| R_{-2}\left(\left.F\right|_{-2} \gamma(\tau)-\zeta h q^{-\frac{1}{h}}\right) \mid \\
\leq & \frac{4 h}{6} \sum_{\ell=1}^{\infty} \ell\left|b_{1,-2,6}(\ell, v)\right| \cdot|q|^{\frac{\ell}{h}}+\frac{2 h}{6 \pi v} \sum_{\ell=1}^{\infty}\left|b_{2,-1,6}(\ell, v)\right| \cdot|q|^{\frac{\ell}{h}} \\
\leq & \frac{2 h}{3} \sum_{\ell=1}^{\infty} 12 \pi \ell^{-\frac{1}{2}}\left[\frac{\ell^{\frac{1}{4}}}{\sqrt{3}} \exp \left(\frac{2 \pi \sqrt{\ell}}{3}\right)+\frac{\pi^{3}}{81} \ell^{\frac{3}{2}} \zeta(3)\right] e^{-\frac{\pi \sqrt{3} \ell}{h}} \\
& +\frac{2 h}{3 \pi \sqrt{3}}\left(\frac{2}{27} \pi^{4} \zeta(3)+\sum_{\ell=1}^{\infty} 12 \pi \ell^{-\frac{3}{2}}\left[\frac{\ell^{\frac{1}{4}}}{\sqrt{3}} \exp \left(\frac{2 \pi \sqrt{\ell}}{3}\right)+\frac{\pi^{3}}{81} \ell^{\frac{3}{2}} \zeta(3)\right] e^{-\frac{\pi \sqrt{3} \ell}{h}}\right)
\end{aligned}
$$

We can clearly estimate this last expression from above by setting $h=6$, which we now do. It is easy to estimate the remaining infinite series in (3.9) by geometric series to obtain our asserted bound $\kappa$.

Remark 3.4. It should be pointed out that in Theorem 5.1.2 in [21] cited in the proof above, a different type of Poincaré series is considered and also in the formulation of the theorem, only actions of matrices in $\mathrm{SL}_{2}(\mathbb{Z})$ rather than Atkin-Lehner involutions on Poincaré series are studied. However, a close inspection of the proof reveals that the result is directly applicable to the situation of the Maass-Poincaré series $P_{1,-2,6}$ acted upon by the Atkin-Lehner involution $W_{d}, d=2,3,6$.

We are now in position to prove our main result, Theorem 1.1.

Proof of Theorem 1.1. Let $Q(x, y)=a x^{2}+b x y+c y^{2}=:[a, b, c]$ be a $\mathrm{SL}_{2}(\mathbb{Z})$-reduced quadratic form of discriminant $\delta=-24 n+1$, i.e., $b^{2}-4 a c=\delta,|b| \leq a \leq c$, and $b>0$ whenever $|b|=a$ or $a=c$. Then exactly one matrix $\gamma_{Q}$ from those 12 in (3.1) satisfies $Q \circ \gamma_{Q}^{-1} \in \mathcal{P}_{\delta}$, see Lemma 3 and Table 1 in [5]. Further define the number $h_{Q} \in\{1,2,3,6\}$ and the 6 th root of unity $\zeta_{Q}$ by the relation

$$
\left.F\right|_{-2} \gamma_{Q}(\tau)=h_{Q} \zeta_{Q} q^{-\frac{1}{h_{Q}}}+O(1)
$$

Then one easily observes that $a \cdot h_{Q} \equiv 0(\bmod 6)$ holds in all possible cases. We focus on the case $a \cdot h_{Q}=12$. Tables 1 and 2 list the quadratic forms $Q$ together with the associated data $\gamma_{Q}, h_{Q}$, and $\zeta_{Q}$, which are easily read off Table 1 in [5] and (3.2)-(3.4).

Now for $\tau_{Q}=-\frac{b}{2 a}+\frac{\sqrt{24 n-1}}{2 a} i$ we find, by Lemma 3.3, that

$$
|P|_{0} \gamma_{Q}\left(\tau_{Q}\right) \mid \in\left(M\left(n ; a, h_{Q}\right)-\kappa, M\left(n ; a, h_{Q}\right)+\kappa\right)
$$

Table 1 Quadratic forms with $a \cdot h_{Q}=12$ for $n$ even

\begin{tabular}{lllll}
\hline $\mathbf{Q}$ & $\boldsymbol{\gamma}_{\mathbf{Q}}$ & $\boldsymbol{h}_{\mathbf{Q}}$ & $\zeta_{\mathbf{Q}}$ & $\boldsymbol{\varphi}_{\mathbf{Q}}$ \\
\hline$[2,-1,3 n]$ & $\gamma_{0,0}$ & 6 & 1 & $-\frac{\pi}{12}$ \\
{$\left[4,1, \frac{3 n}{2}\right]$} & $\gamma_{\frac{1}{2}, 1}$ & 3 & $\zeta_{6}$ & $\frac{5 \pi}{12}$ \\
{$[6,-5, n+1]$} & $\gamma_{\frac{1}{3}, 0}$ & 2 & 1 & $-\frac{5 \pi}{12}$ \\
{$\left[12,1, \frac{n}{2}\right]$} & $\gamma_{\infty}$ & 1 & 1 & $\frac{\pi}{12}$ \\
\hline
\end{tabular}


Table 2 Quadratic forms with $a \cdot h_{Q}=12$ for $n$ odd

\begin{tabular}{lllll}
\hline $\mathbf{Q}$ & $\boldsymbol{\gamma}_{\mathbf{Q}}$ & $\boldsymbol{h}_{\mathbf{Q}}$ & $\boldsymbol{\zeta}_{\mathbf{Q}}$ & $\boldsymbol{\varphi}_{\mathbf{Q}}$ \\
\hline$[2,-1,3 n]$ & $\gamma_{0,3}$ & 6 & -1 & $\frac{11 \pi}{12}$ \\
{$\left[4,-3, \frac{3 n+1}{2}\right]$} & $\gamma_{\frac{1}{2}, 2}$ & 3 & $\zeta_{6}^{-1}$ & $-\frac{7 \pi}{12}$ \\
{$[6,-5, n+1]$} & $\gamma_{\frac{1}{3}, 1}$ & 2 & -1 & $\frac{7 \pi}{12}$ \\
\hline
\end{tabular}

and

$$
\arg \left(P_{0} \gamma_{Q}\left(\tau_{Q}\right)\right) \in\left(\varphi_{Q}-\arctan \left(\frac{\kappa}{M\left(n ; a, h_{Q}\right)}\right), \varphi_{Q}+\arctan \left(\frac{\kappa}{M\left(n ; a, h_{Q}\right)}\right)\right),
$$

where

$$
M\left(n ; a, h_{Q}\right):=\left(1-\frac{2 a h_{Q}}{2 \pi \sqrt{24 n-1}}\right) e^{2 \pi \frac{\sqrt{24 n-1}}{2 a h_{Q}}}
$$

and $\varphi_{Q}$ as given in Tables 1 and 2 denotes the argument of the main term of $\left.P\right|_{0} \gamma_{Q}\left(\tau_{Q}\right)$, which is given by $\arg \left(\zeta_{Q}\right)+\frac{b \pi}{12}$. If we now assume that $n \geq 54$, then we see that there cannot be any singular moduli with $a \cdot h_{Q} \neq 12$ matching those with $a \cdot h_{Q}=12$ because the difference

$$
\left|\left(1-\frac{2 a_{1} h_{Q_{1}}}{2 \pi \sqrt{24 n-1}}\right) e^{2 \pi \frac{\sqrt{24 n-1}}{2 a_{1} h Q_{1}}}-\left(1-\frac{2 a_{2} h_{Q_{2}}}{2 \pi \sqrt{24 n-1}}\right) e^{2 \pi \frac{\sqrt{24 n-1}}{2 a_{2} h} Q_{2}}\right|
$$

would be larger than $2 \kappa$ if $a_{1} h_{Q_{1}}=12$ and $a_{2} h_{Q_{2}} \neq 12$. The same is true for the distortion in the arguments among those which have $a \cdot h_{Q}=12$, since for $n \geq 54$ we have

$$
\left|\arctan \left(\frac{\kappa}{M\left(n ; a, h_{Q}\right)}\right)\right| \leq \frac{\pi}{24},
$$

so in particular less than half of the smallest possible difference between two distinct $\varphi_{Q}$ from Tables 1 and 2 . Hence all these values can only be simple zeros of $\widehat{H}_{\delta}$ and by Proposition 3.2, the theorem is proven for $n \geq 54$. The remaining cases are checked by comparing with numerically computed examples ${ }^{\mathrm{b}}$.

\section{Endnotes}

${ }^{a}$ From here on, the term "quadratic form" always means "positive definite integral binary quadratic form" if not declared otherwise.

b In particular, we refer the reader to Sutherland's extensive table at http://math.mit. $\mathrm{edu} / \sim \mathrm{drew} /$ Pfiles/ for our test of the small cases.

\section{Competing interests}

The authors declare that they have no competing interests.

Authors' contributions

Both authors drafted and wrote the manuscript jointly. Both authors have read and approved the final manuscript.

\section{Acknowledgements}

The authors are grateful to Kathrin Bringmann and Andrew Sutherland for many useful conversations and comments which greatly improved the exposition of this paper. They would also like to thank the anonymous referee for helpful comments. The research of the first author leading to these results has received funding from the European Research Council under the European Union's Seventh Framework Programme (FP/2007-2013) / ERC Grant agreement n. 335220 AQSER. The second author thanks the University of Cologne and the DFG for his generous support via the University of Cologne postdoc grant DFG Grant D-72133-G-403-151001011. 


\section{References}

1. Bringmann, K, Ono, K: Coefficients of harmonic Maass forms. In: Proceedings of the 2008 University of Florida Conference on Partitions, q-series, and modular forms

2. Bringmann, K, Ono, K: An arithmetic formula for the partition function. Proc. Amer. Math. Soc. 135, 3507-3514 (2007)

3. Bruinier, JH, Ono, K: Algebraic formulas for the coefficients of half-integral weight harmonic weak Maass forms. Adv. Math. 246, 198-219(2013)

4. Bruinier, JH, Ono, K, Sutherland, AV: Class polynomials for nonholomorphic modular functions. preprint

5. Dewar, M, Murty, MR: A derivation of the Hardy-Ramanujan formula from an arithmetic formula. Proc. Amer. Math. Soc. 141(6), 1903-1911 (2013)

6. Euler, L: Evolutio producti infiniti $(1-x)(1-x x)\left(1-x^{3}\right)\left(1-x^{4}\right)\left(1-x^{5}\right)\left(1-x^{6}\right)$ etc. Opera Omnia. 1(3), 472-479

7. Fay, JD: Fourier coefficients of the resolvent for a Fuchsian group. J. Reine Angew. Math. 293/294, 143-203 (1977)

8. Folsom, A, Masri, R: Equidistribution of Heegner points and the partition function. Math. Ann. 226, 3724-3759 (2011)

9. Gross, B, Kohnen, W, Zagier, D: Heegner points and derivatives of $L$-series II. Math. Ann. 278, 497-562 (1987)

10. Gradsteyn, IS, Ryzhik, IM: Table of Integrals, Series, and Products. 6th ed. Academic Press (2000)

11. Hardy, GH, Ramanujan, S: Asymptotic formulae in combinatory analysis. Proc. London Math. Soc. 17(2), 75-115 (1918)

12. Hejhal, DA: The Selberg trace formula for $\operatorname{PSL}(2, \mathbb{R})$, Springer Lect. Notes in Math, Vol. 1001. Springer-Verlag, Berlin (1983)

13. Koecher, M, Krieg, A: Elliptische Funktionen und Modulformen. 1st ed. Springer-Verlag (1998)

14. Lang, S: Elliptic functions. 2nd edition, Graduate Texts in Mathematics, Vol. 112. Springer-Verlag, New York (1987)

15. Lee, P, Zamorzaev, A: Parity of the partition function and traces of singular moduli. Int. J. Number Theory. 8, 395-409 (2012)

16. Larson, E, Rolen, L: Integrality Properties of the CM-values of Certain Weak Maass Forms, Forum Mathematicum (2013). doi:10.1515/forum-2012-0112

17. Luke, YL: Inequalities for generalized hypergeometric functions. J. Approx. Theory. 5, 41-65 (1972)

18. Masser, D: Elliptic Functions and Transcendence. Lecture Notes in Mathematics, Vol. 437. Springer-Verlag (1975)

19. Ono, K: Unearthing the visions of a master: harmonic Maass forms and number theory. Curr Dev Math. 2008 347-454 (2009)

20. Rademacher, $\mathrm{H}$ : On the partition function $p(n)$. Proc. London Math. Soc. 43(2), 241-254 (1937)

21. Rankin, RA: Modular forms and functions. Cambridge University Press (1977)

22. Schertz, R: Weber's class invariants revisited. J. Théor. Nombres Bordeaux. 14(1), 325-343 (2002)

\section{Submit your manuscript to a SpringerOpen ${ }^{\circ}$ journal and benefit from:}

- Convenient online submission

- Rigorous peer review

- Immediate publication on acceptance

- Open access: articles freely available online

- High visibility within the field

- Retaining the copyright to your article

Submit your next manuscript at $\mathbf{s p r i n g e r o p e n . c o m ~}$ 